\title{
Synthesis and Characterization of Pure Cobalt Ferrite for DC Electrical
}

\author{
Rashmi Kulshrestha, Vinay Anand
}

\begin{abstract}
Ferrite Cobalt $\left(\mathrm{CoFe}_{2} \mathrm{O}_{4}\right)$ is a distinguished magnetic material with average enforcement and average magnitude of magnetization. It has a distinct chemical stability and mechanized hardness. It is an expectant advocate for the procedure of sensory devices and actuators, as a definitive sealing, magnetic drug target, and electrical devices and has an extensive range of research in material technology/science for technological uses/applications. The naoparticles of cobalt ferrite on an entangled effectively processed at ambient temperature through a simple co-precipitation process. The crystal structure \& morphology of symbol was determined by XRD and SEM. The $X R D$ spectrum confirms that the composite nanoparticles are formed by the perfect spinel structure. The average rate of crystals was determined by means of Modified Scherer (54nm) and Williamson-Hall (49nm) methods. A SEM view showed the nanoparticles $\mathrm{CoFe}_{2} \mathrm{O}_{4}$ being grouped in nearby structures. The bond and visual features were described by FTIR and UV-Vis Spectrum. Compared with other absorbing bands informed in literature, this material shows a very large intake band between 350 and $600 \mathrm{~cm}-1$ in the FTIR test. More than one pitch in $U V$. Vis spectrum is seen with a direct straight band gap identified of $4.1 \mathrm{eV}$ and $4.9 \mathrm{eV}$. This is again in contrast to other reports in literature. These irregular outcomes express that although a very good spinel structure is in place, coordination of the symbols in the cells of the unit is different here. An electronic DC training for the temperatures cobalt ferrite was tested for temperature, which again showed some interesting results from those reported in literature. This combination of irregular buildings in considerable degree of the ferrite needs to demonstrate a deeper analysis of the overall structure and characteristics of cobalt ferrite in comparison to these methods.
\end{abstract}

Keywords: Cobalt ferrite; magnetic nanoparticles; dc electrical study; spinel structure.

\section{INTRODUCTION}

Magnetic ferrites with a spinel structure have attracted the attention of the global marketplace and many of their researchers have produced their remarkable and amazing electromagnetic features. They are easily prepared and the strength of different conditions used in technological industry. The changes that occur in the physical, chemical, electrical and magnetic properties through changing dimensions of pieces have inspired researchers around the world to make these materials with new buildings. The magnetic, electronic and magneto-practical features at the cobalt-rich cobalt and top-rise feather have begun to be the most used ferrite system in magnetic recording and magnetic resin making devices. Spinel ferrite, cobalt ferrite $\left(\mathrm{CoFe}_{2} \mathrm{O}_{4}\right)$ spinel interfaces with an insensitive spinel structure include the most suitable magnetic materials for the high-crystalline -anisotropy, encryption of average size, high temperature, decent electrical properties, respectable mechanical features and chemical stability [1]-[5].

The properties and all these features of cobalt ferrite are suitable components for sensory devices and actuators, shaft seals, space warrants, electrical appliances, data storage, magnetic drug concentrations, biosensor, and magnesium freezing, materials research, optics, art and making something amazing, good core source for power movements in electricity and electronic industry, registration ends, the antenna rods, loading coils and telecommunications applications [6]-[11].

Interpretation of dimensions of ferrites to nanometer scale can be achieved through an inclusive range of methods, physical and chemical. The physical methods where powder of mass precursor materials (high priority) are converted to Nano-crystalline particles with mechanical, metallic or laser processes, this process is termed as a "top-down systems". Conversely, chemical systems are based on solutions of "bottom-up" systems where nanoparticles are combined by chemical reactions between sub-nanometer subspecies, such as atoms/molecules [7], [13].

While the simple and applied physical methods of processing, allow for a comprehensive series of ferrite Nano-powder to be measured in large proportions and at relatively low cost, the reliability is often questioned because of contamination of the products (especially in the mechanical milling processes) and limited control of the morphology of Nano/micro sized materials that come out The wet chemical artificial trails have been more profitable for preparation/manufacturing of transition metal ferrites than physical means since they give more control over the size, extent of distribution, shape and level of the collections of nanocrystals, created. Chemical sharing is the most common method of production for its high yield and simplicity in the preparation/manufacturing of ultrafine magnetic nanocrystals [9], [10], [12].

Ferrites are mixed/blend oxides with the general $\mathrm{AB}_{2} \mathrm{O}_{4}$ formula, wherever $\mathrm{A}$ is a splitting of natural metal $\& \mathrm{~B}$ is the trivalent ferric ion. They have a backbone structure (spinal type) similar to that of a mineral spinel, $\mathrm{MgAl}_{2} \mathrm{O}_{4}$. In a typical spinel lattice, the voluminous oxide ions $\left(\mathrm{r}_{0}{ }^{2}-=124\right.$ pm) take the closed arrangement with cubes, thus creating two different types, i.e. tetrahedral (A) and octahedral (B).
Revised Manuscript Received on September 14, 2019

Dr.Rashmi Kulshrestha, Department of Chemistry, Sanskriti University, UttarPradesh, India.(E-mail: sanpubip@gmail.com)

Vinay Anand, Department of Electrical Engineering, Sanskriti University, UttarPradesh, India.(E-mail: sanpubip@gmail.com) 


\section{EXPERIMENTAL METHOD}

\section{Materials}

All the materials used were of reagent rating \& were further used without purification. Double-natured water was used as manure. "Ferric nitrate $(\mathrm{Fe}(\mathrm{NO} 3) 3.9 \mathrm{H} 2 \mathrm{O},>98 \%$ pure), cobalt nitrate $\left(\mathrm{Co}\left(\mathrm{NO}_{3}\right)_{2} \cdot 6 \mathrm{H}_{2} \mathrm{O},>99 \%\right.$ pure $)$ and ammonia solution $(25 \% \mathrm{w} / \mathrm{v})$ of HPLC grade were used. Oleic acid of HPLC grade was employed as a surfactant".

\section{Procedure}

The same amount of $2 \mathrm{M}$ ferric nitrate soln \& $1 \mathrm{M}$ nitrate cobalt solution was prepared in double, de-ionized and motion water after mixing, has stirred attractive for 15 minutes. To avoid the result of barrels, the metal salt solution was prepared in highly ionized distilled water. $4 \mathrm{M}$ ammonia resolution was manufactured/prepared \& slowly slipped down above for salt. The solution of the solution $\mathrm{pH}$ was checked regularly as the ammonia solution was additionally present. The above materials were regularly worn using magnetic mesh to the extent of $\mathrm{pH}$ of 9-10. A fixed size of oleic acid was mixed to solution as surfactant material and coat material. Water was then heated to temperature of $80^{\circ} \mathrm{C}$ and immersed for an hour.

Then the products were cooled to hotness of a room. To remove pieces of chemicals from ammonia, nitrogen or salt, the precipitate was then washed twice with distilled water and then remove the insurance from solution washed with ethanol. To separate the different juice, the rhyme was then issued for twenty minutes at $5000 \mathrm{rpm}$. The extra rain was removed, and the remaining material was scrapped again, until a little black cream was left. Then the precipitate was dried at night at $70^{\circ} \mathrm{C}$. The sample was then buried to a fine/ powder and released at $1000^{\circ} \mathrm{C}$ for four hours.

\section{CHARACTERISATION RESULTS}

\section{XRD Analysis}

The X-Ray Extension (XRD) of the sample was made using X-Ray Diffractometer (RIGAKU LAB SMART) with monochromatic $\mathrm{Cu}-\mathrm{K} \alpha$, wavelength $1.5418 \AA$ at $40 \mathrm{KV} \&$ $30 \mathrm{~mA}$. For the standard piece, the composite cobalt ferrite powder has no additional degree. Figure 1 shows the XRD pattern of $\mathrm{CoFe} 2 \mathrm{O} 4$. These patterns confirm the creation of a cubic grid (fd-3m interplanetary group) which corresponds well with JCPDS No. 22-1086 [14].

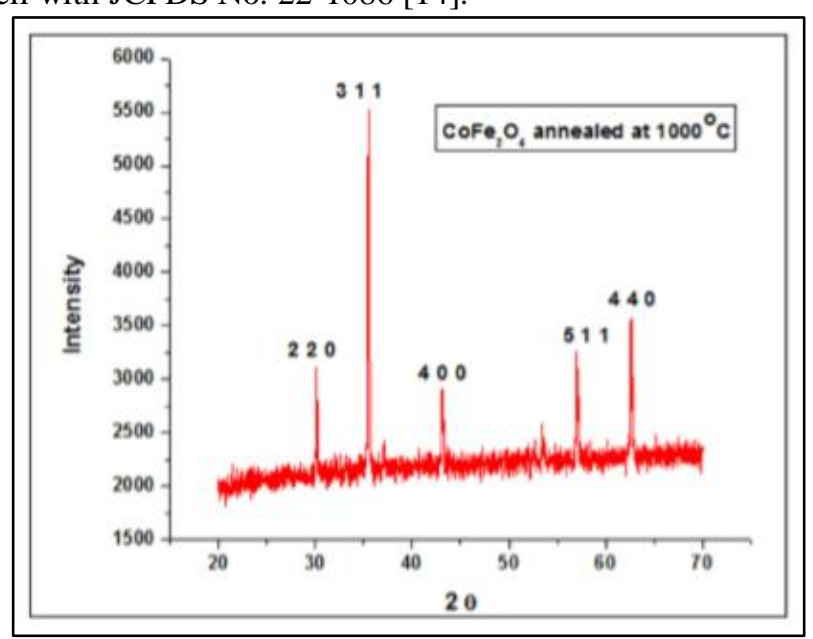

Figure 1. XRD of $\mathrm{CoFe2O4}$
The Scherer Modified parts Figure 2 and WilliamsonHall Figure 3 were also drawn and the mean proportions of average crystals as measured at $54 \mathrm{~nm}$ and $49 \mathrm{~nm}$, with the latter having a lattice strain of 0.001110 .

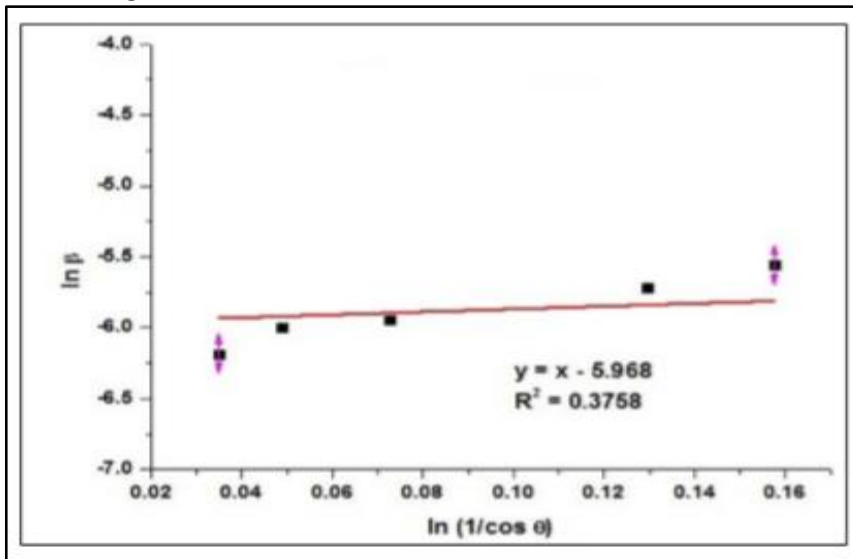

Figure 2. Modified Scherrer plot of $\mathrm{CoFe2O4}$

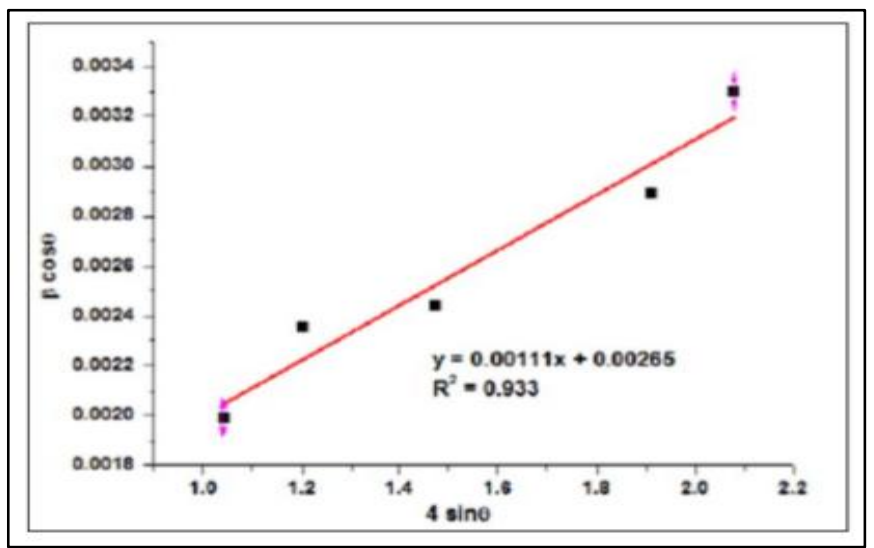

Figure 3. Williamson-Hall plot of $\mathrm{CoFe2O4}$ SEM Analysis

A sample (microstructural) prepared by JSM6010PLUS_LA with fast-speed voltage was assessed from 0.5-20 kV. The SEM default image of the $\mathrm{CoFe}_{2} \mathrm{O}_{4}$ sample is released at $1000^{\circ} \mathrm{C}$ for 4 hours appear in Figure 4. It can be seen that there is no sign of pollution in the samples. The small pieces are virtually spiral in shape and may have the added benefit of reinforcing the magical properties of the pieces.

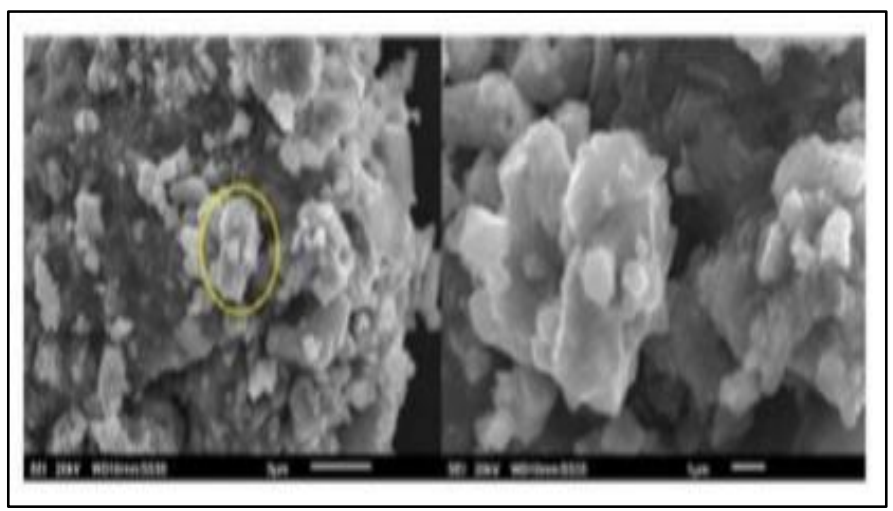

Figure 4. SEM figure of $\mathrm{CoFe2O} 4$ nanoparticles annealed at $10000 \mathrm{C}$

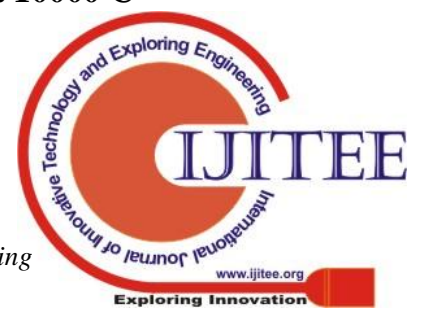




\section{FTIR Analysis}

Perkin Elmer Frontier FTIR Spectrometer with a $\mathrm{KBr}$ device and the $\mathrm{KBr}$ device have been used for FTIR analysis. A spectrum was found with the wave count going between $400 \mathrm{~cm}^{-1}$ to $4500 \mathrm{~cm}^{-1}$. The standard FT-IR scale for the sample pulled back at $1000^{\circ} \mathrm{C}$ is exposed in Figure 5. The spectacle indicates that in the range 300 to $800 \mathrm{~cm}^{-1}$, the band contains impenetrable bonds that are a standard feature of the ferrite spinel.

The highest frequency incidence band ( $v 1)$ lies in the range of between 500 and $600 \mathrm{~cm}^{-1}$ and is set for the incised metal-fettered metal plate, which includes a link between the oxide ion and the metal ion of a tetrahedral site $\left(\mathrm{O}-\mathrm{M}_{\mathrm{Tet}}\right)$, and the most usual inlet (v2) lies between 400 and $490 \mathrm{~cm}^{-1}$ and is positioned for the vibrating metal octahedral complex. Including the oxide link with metal ion of an octahedral site $\left(\mathrm{O}-\mathrm{M}_{\mathrm{Oct}}\right)$. The frequency $v$ band is often caused by stretching within distribution between molecules / ion bands, and the minor frequency band is result of shaking. These collective scenarios are in good condition with the usual luxury synthetic bands of cobalt ferrite nanocrystals [15].

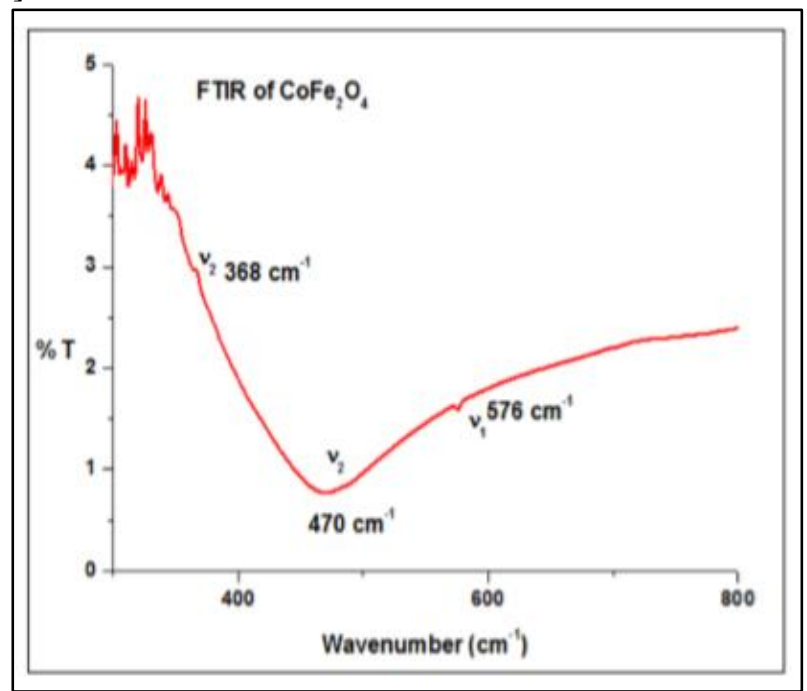

Figure 5. FTIR spectra of $\mathrm{CoFe} 2 \mathrm{O} 4$ sample annealed at $1000{ }^{\circ} \mathrm{C}$ for 4 hours

\section{UV-Vis Analysis}

The UV-jet spectrum was removed by Perkin Elmer's spectrophotometer. UV-Visible sensation of nanoparticles $\mathrm{CoFe} 2 \mathrm{O} 4$ is presented in Figure 6. From Fig it is found that there is tail in a complex spectrum of $\mathrm{CoFe}_{2} \mathrm{O}_{4}$ nanoparticles in the visible area. However, in the UV field that a tail was not seen like that. In addition, the absorbing spectroscopy has an important role in determining a band gap that can be measured from Tauc's relationship,

$$
\alpha h v=A(h v-E g)^{n}
$$

where, Eg is energy gap, constant $A$, is different for diverse transitions, $(\mathrm{h} v)$ is energy of incident photon and $\mathrm{n}$ is a constant value, which is equal to 2 for incidental band gap semiconducting materials [16].

Figure 7 shows the plot of $(\alpha h v)^{2}$ against $(h v)$. It has been seen from Figure 7. The aggregate value of the band for the $\mathrm{CoFe}_{2} \mathrm{O}_{4}$ nanartarticles is real to $4.1 \mathrm{eV}$.

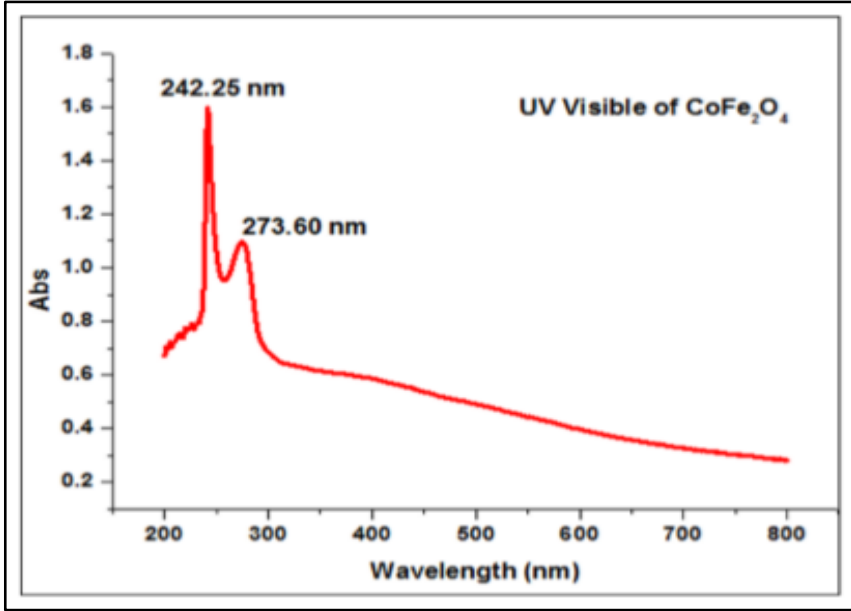

Figure 6. UV-Vis spectrum of $\mathrm{CoFe2O} 4$ nanoparticles

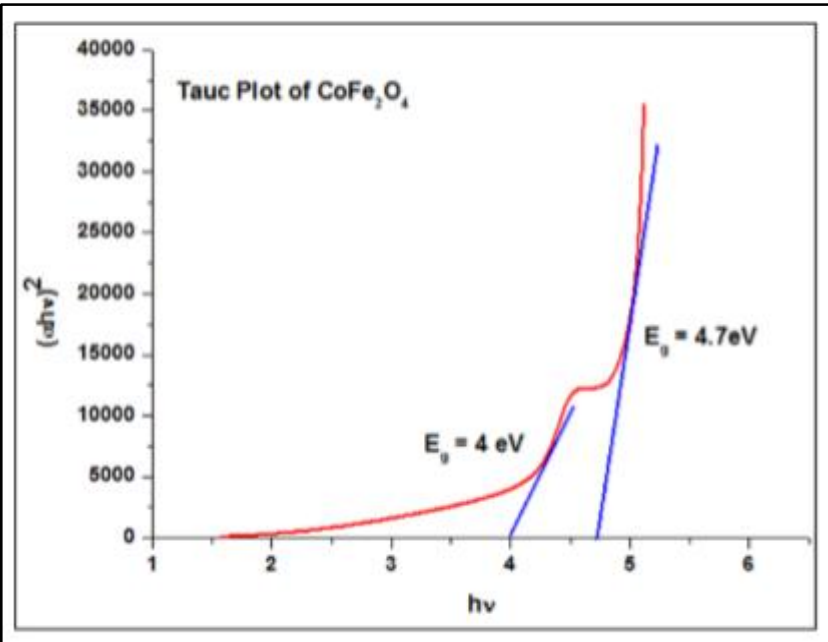

Figure 7. Tauc plot for straight band gap of $\mathrm{CoFe2O4}$ nanoparticles

\begin{tabular}{||c|c|c|}
\hline $\begin{array}{c}\text { SYNTHESIS } \\
\text { METHOD }\end{array}$ & $\begin{array}{c}\text { REPORTED } \\
\text { BANDGAP VALUES }\end{array}$ & REFERENCE \\
\hline Co-precipitation & (direct) $3.85 \mathrm{eV}$ & S. Singh et al. \\
\hline PLD (thin film) & (direct) $2.5 \mathrm{to} 2.6 \mathrm{eV}$ & $\begin{array}{c}\text { A.V.Ravindra, P. } \\
\text { Padhan }\end{array}$ \\
\hline PLD (thin film) & $\begin{array}{c}\text { (direct) } 1.95 \mathrm{eV} \\
\text { (indirect) } 1.42 \mathrm{eV}\end{array}$ & Himcinschi et al. \\
\hline PLD (thin film) & $\begin{array}{c}\text { (direct) } 2.74 \mathrm{eV} \\
\text { (indirect) } 1.18 \mathrm{eV}\end{array}$ & Holinsworth et al. \\
\hline $\begin{array}{c}\text { Electrostatic spray } \\
\text { (thin film) }\end{array}$ & (direct) $2.57 \mathrm{eV}$ & $\begin{array}{c}\text { Rajendra S. } \\
\text { Gaikwad et al. }\end{array}$ \\
\hline \multicolumn{2}{|c|}{ Asokarajan R., } \\
Solvo-thermal & (direct) $1.81 \mathrm{eV}$ & $\begin{array}{c}\text { Neyvasagam K., } \\
\text { Milton Franklin } \\
\text { Benial }\end{array}$ \\
\hline
\end{tabular}

Figure 8. Comparison of reported band gap values 


\section{D.C. ELECTRICAL CONDUCTIVITY OF COFE2O4}

The sample response was analyzed to D.C. the voltage to better identify and understand the harvesting equipment in this material. In this test measure the resilience of the ferrite cobalt sample as a temperature obligation, from about $50{ }^{\circ} \mathrm{C}$ up to $500{ }^{\circ} \mathrm{C}$, using a cryostat with a heating level. The system measures D.C. against different temperatures. The sample is usually bore with mass material designed by a comma and the regular place. Silver / gold covers are made on both margins of the discs to make connections. Differentlevel DC voltage is activated and the output flow is read at the same time at different temperatures.

Figure 9 indicates that DC speed is increasing by means of an upsurge in temperature and concludes the equation of syntax for semiconducting products, i.e.

$$
\sigma_{d c}=\sigma_{0} \exp \left(-\frac{E_{d c}}{k t}\right)
$$

Where, $\sigma_{\mathrm{o}}$ is pre-exponential aspect, which depends on composition, mobility of diffusing ions, etc. and $E_{\mathrm{dc}}$ is the D.C. activation energy. The initiation energy for transmission mechanism / procedure was premeditated from the slope of straight line ration Figure 10. The value of the active energy is obtained to $\mathrm{E}_{\mathrm{dc}}=0.305 \mathrm{eV}$ and the carrying value changes from $10^{-9} \mathrm{~S} / \mathrm{cm}$ to $10^{-6} \mathrm{~S} / \mathrm{cm}$ with an increase in temperature [17], [18].

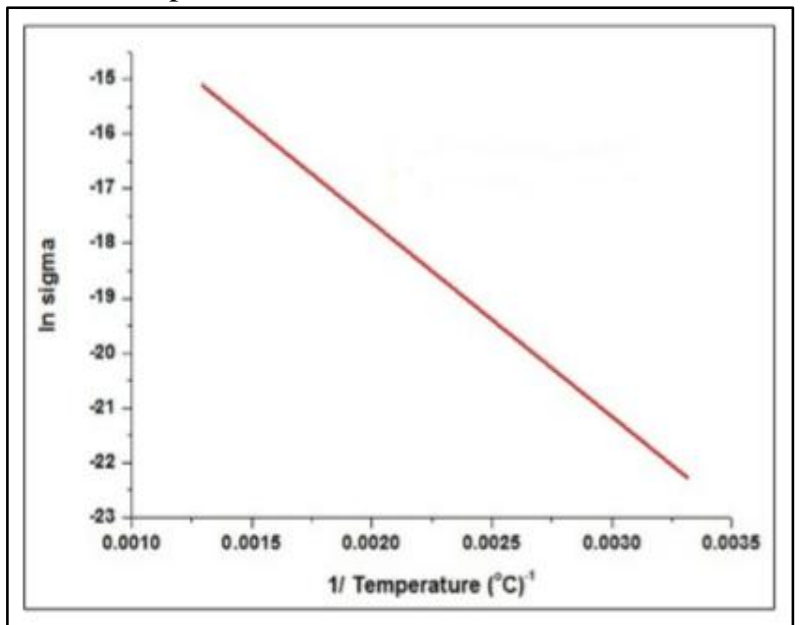

Figure 9. DC conductivity variation with temperature of CoFe2O4 nanoparticles (linear fit)

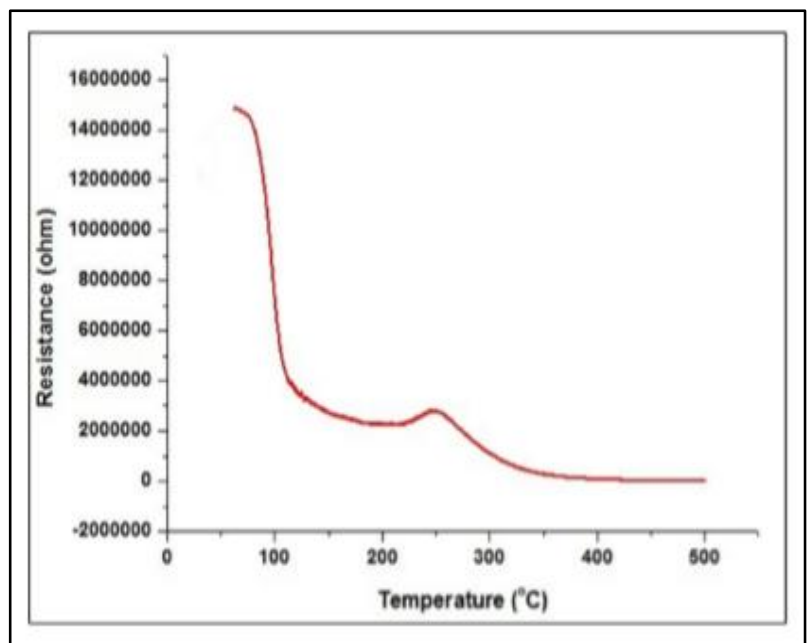

Figure 10. Variation plot of Resistance vs. Temperature

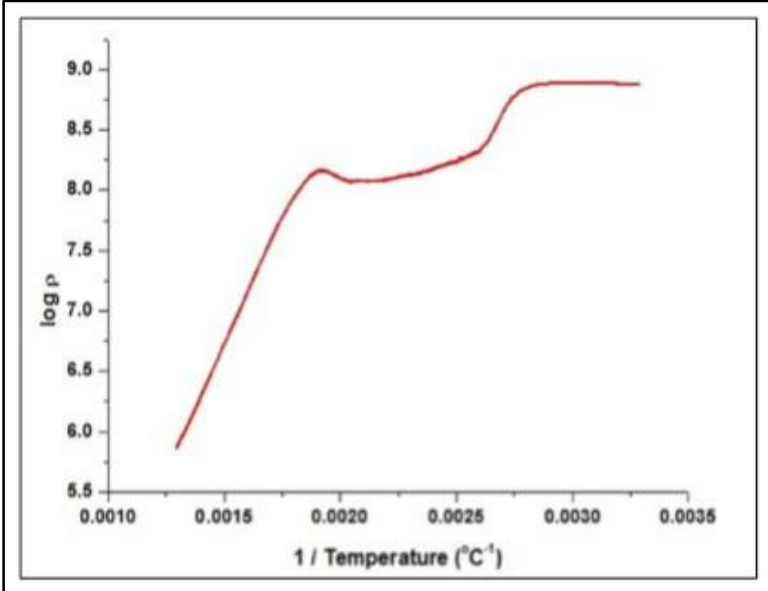

Figure 11. Variation plot of Log $\rho$ vs. Temperature

Ferrites Cobalt is known as semiconducting material, and this is confirmed by the rise in temperature movement for the test tested Figure 9. The charge carrier in Cobalt boats may be $\mathrm{Co}^{2+}, \mathrm{Co}^{3+}, \mathrm{Fe}^{2+}, \mathrm{Fe}^{3+}$ and may all be present in octahedral sites. The electrical movement in ferrites can be defined on the source of the exchange of electricity among ions of identical element that is presently contained in more than one distributed valence state across a corresponding crystallographic lattice sites [19]-[22]. It is recognized that variation in slope is accredited to Curie temperature or to change in the distribution device. At Curie's temperature, its sampling transformation from a ferrimagnetic state is conditioned to an impaired paramagnetic state with a substantial increase in behavior. The time of $520 \mathrm{~K}$ time may be an outcome of movement from ferromagnetic to paramagnetic nature [23].

\section{CONCLUSION}

Nanoparticles ferrite cobalt were successfully synthesized refined through a chemical sharing system at environmental temperature. The XRD demonstrates that the maximum density was $2 \Theta=35.48^{\circ}$, which is the same as the reflective plane (3-1-1). The average sizes of particle measured by the Scherer and Williamson-Hall methods are $54 \mathrm{~nm}$ and $49 \mathrm{~m}$ respectively. The results of SEM show that items have gone up in nearby structures and possibly will have been composed as a result of the magical nature of the pieces. FTIR investigations for cobalt ferrite nanoparticles show a very broad bandwidth between 350 and $600 \mathrm{~cm}^{-1}$. v1 ("vibration of the tetrahedral metal complex") lies between 500 and $600 \mathrm{~cm}^{-1}$, and $v 2$ ("vibration of the octahedral metal complex") lies between 400 and $490 \mathrm{~cm}^{-1}$. Further than one peak is visible. The UV-Vis spectrum with a direct vertical gap and 4.1 and $4.9 \mathrm{eV}$. These irregular results show a very high standard of structure; coordination of dressings, grain size and synchronization method can affect the band's gap. Finally, the electrical study showed DC resistivity decreased in value by increasing temperatures, and therefore affects automatic nature. It is also seen that the synoptic nanoparticles in undergo a phase transition around $520 \mathrm{~K}$, according to the zig-zag/curie temperature.

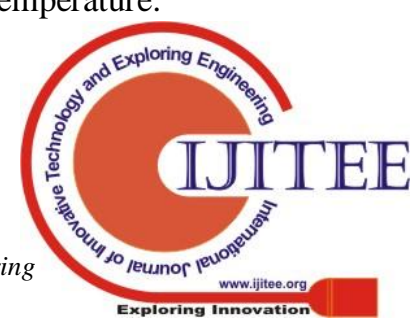




\section{REFERENCE}

1. F. Cardarelli, "Magnetic Materials," in Materials Handbook, London: Springer London, 2000, pp. 275290.

2. N. N. Greenwood and A. Earnshaw, "Chemistry of the Elements Second Edition."

3. M. Sugimoto, "The Past, Present, and Future of Ferrites," J. Am. Ceram. Soc., vol. 82, no. 2, pp. 269-280, Dec. 2004.

4. D. Caruntu, G. Caruntu, and C. J. O'Connor, "Magnetic properties of variable-sized $\mathrm{Fe}_{3} \mathrm{O}_{4}$ nanoparticles synthesized from non-aqueous homogeneous solutions of polyols," J. Phys. D. Appl. Phys., vol. 40, no. 19, pp. 5801-5809, Oct. 2007.

5. V. L. Calero-DdelC and C. Rinaldi, "Synthesis and magnetic characterization of cobalt-substituted ferrite (CoxFe3-xO4) nanoparticles," J. Magn. Magn. Mater., vol. 314, no. 1, pp. 60-67, Jul. 2007.

6. R. V. Kumar, Y. Koltypin, X. N. Xu, Y. Yeshurun, A. Gedanken, and I. Felner, "Fabrication of magnetite nanorods by ultrasound irradiation," J. Appl. Phys., vol. 89, no. 11, pp. 6324-6328, Jun. 2001.

7. C. R. Hammond, "THE ELEMENTS."

8. M. Pinzon, "Introduction to Magnetism and Magnetic Materials."

9. D. Jiles, "Introduction to Magnetism and Magnetic Materials Second edition."

10. "Chapter 1 Magnetic Materials."

11. R. C. O'Handley, Modern magnetic materials: principles and applications. Wiley, 2000.

12. A. Mandal, "Size-controlled synthesis of magnetite nanoparticles in the presence of polyelectrolytes," Chem. Mater.

13. R. Lawaczeck, M. Menzel, and H. Pietsch, "Superparamagnetic iron oxide particles: contrast media for magnetic resonance imaging," Appl. Organomet. Chem., vol. 18, no. 10, pp. 506-513, Oct. 2004.

14. B. D. Cullity, "Elements of X-ray diffraction, 2nd edition," Addison-Wesley Publ. Co. Read. MA, 1978.

15. V. Kumar, A. Rana, N. Kumar, and R. P. Pant, "Investigations on controlled-size-precipitated cobalt ferrite nanoparticles," Int. J. Appl. Ceram. Technol., 2011.

16. M. Żenkiewicz and J. Richert, "Influence of polymer samples preparation procedure on their mechanical properties," 2008

17. Modern Ferrite Technology. 2006.

18. "Microwave Materials."

19. K. K. Bamzai, G. Kour, B. Kaur, and S. D. Kulkarni, "Preparation, and Structural and Magnetic Properties of Ca Substituted Magnesium Ferrite with Composition $\mathrm{MgCa}_{x} \mathrm{Fe}_{2-x} \mathrm{O}_{4}(\mathrm{x}=0.00,0.01,0.03,0.05$ 0.07)," J. Mater., vol. 2014, pp. 1-8, Apr. 2014.

20. K. K. Bamzai, G. Kour, B. Kaur, S. D. Kulkarni, and C. M. Julien, "Preparation, and Structural and Magnetic Properties of $\mathrm{Ca}$ Substituted Magnesium Ferrite with Composition $\mathrm{MgCa}$ x Fe 2-x O 4 (í $\mu$ í士¥ = 0.00, 0.01, $0.03,0.05,0.07), " 2014$.

21. S. S. Khot, N. S. Shinde, B. P. Ladgaonkar, B. B. Kale, and S. C. Watawe, "Effect of Temperature of Synthesis on X-Ray, Infrared Spectroscopic Study and Magnetic Properties of Magnesium Zinc Ferrites," Key Eng. Mater., vol. 547, pp. 57-69, Apr. 2013.

22. H. K. Dubey, C. Verma, S. Rai, A. Kumar, and P. Lahiri, "Notes Synthesis, characterization and properties of nickel based zinc ferrite nanoparticles," 2019.

23. A. A. Sattar, "Temperature Dependence of the Electrical Resistivity and Thermoelectric Power of Rare Earth Substituted Cu-Cd ferrite," 2003. 